\title{
A Study on Subjectivities of Type 1 and 2 in Parameters of Differential Equations
}

\author{
R.M. JAFELICE ${ }^{1 *}$, A.M. BERTONE ${ }^{1}$ and R.C. BASSANEZI ${ }^{2}$
}

Received on November 29, 2013 / Accepted on January 27, 2015

\begin{abstract}
This study presents the Verhulst's model for the analysis of population growth with the rate of reproductivity depending on the fertility rate and the country economic development. These linguistic variables are defined through Fuzzy Rule-Based Systems (FRBS). The analysis is made for FRBS types 1 and 2 where in the first case, the inference method used is Mamdani's and the defuzzification is the center of gravity. For type-2 FRBS is used as input variables interval type-2 fuzzy sets, and as output intervals. The output is defuzzificated by the Type Reducer method that use the algorithm of Karnik-Mendel (KM). The aim of this study is to compare the solutions of the Verhulst's model where the parameter, rate of reproductivity, is determined through the type- 1 and type-2 FRBS. The comparison is made computing the region built from the solutions corresponding to the minimum and maximum rate. It has been noticed that the region corresponding to type-2 FRBS is contained in the region built similarly from type-1 FRBS, showing a higher accuracy in the response [1], [2] and [4].
\end{abstract}

Keywords: fuzzy sets, fuzzy rule-based systems, ordinary differential equations.

\section{INTRODUCTION}

In his pioneering work [8] Zadeh proposed an extension of classical fuzzy sets for the concept of type-2 fuzzy sets, which would be especially useful in those situations where exact membership function for a fuzzy set is difficult to determine. This theory allows the modeling of the linguistic type, such as FRBS, minimizing the effects of uncertainties [4]. The classic fuzzy sets started to be known as type-1. Since the assignment of a precise number for the expert opinion is too restrictive for the analysis of real models, the type-2 fuzzy sets appear as better tools to cope with uncertainty or gradual data. The main difference between both types is due to the fact that the membership functions or type-2 fuzzy sets are themselves fuzzy sets. On the other hand, these sets are currently the most widely used due to their reduced computational cost.

Let $X$ be an universe. By definition, a type-1 fuzzy subset $A \subset X$ is given by $\mu_{A}: X \rightarrow[0,1]$; $\mu_{A}$ is called the membership function. The value $\mu_{A}(x)$ represents the degree of membership of

*Corresponding author: Ana Maria Amarillo Bertone

${ }^{1}$ FAMAT, UFU, Uberlandia, MG, Brazil. E-mails: rmotta@ufu.br; anamaria@ famat.ufu.br

${ }^{2}$ UNICAMP, Campinas, SP, Brazil. E-mail: rodney@ime.unicamp.br 
$x \in A$. A type-2 fuzzy set, $\widetilde{A}$ in $X$, is characterized by its membership function, $\mu_{\widetilde{A}}: X \times J_{x} \rightarrow$ $[0,1]$, where $J_{x} \subset[0,1]$ is an interval for each $x \in X$. In particular, when $\mu_{\widetilde{A}}(x, u)=1$ for all $u \in J_{x}$ and $x \in X$, the fuzzy set is called interval type- 2 fuzzy set. Figures 1 and 2 show the two types of fuzzy sets.

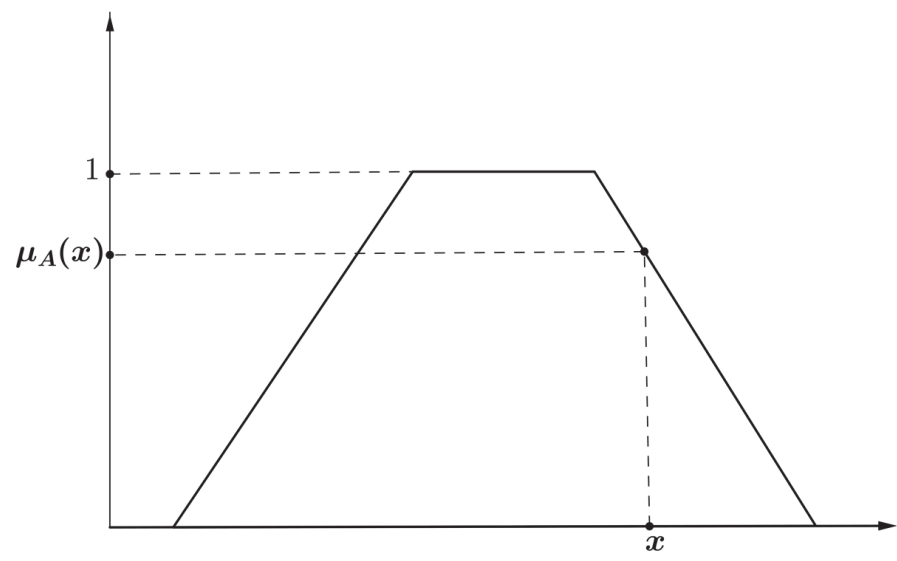

Figure 1: Classic fuzzy set or type-1 fuzzy set.

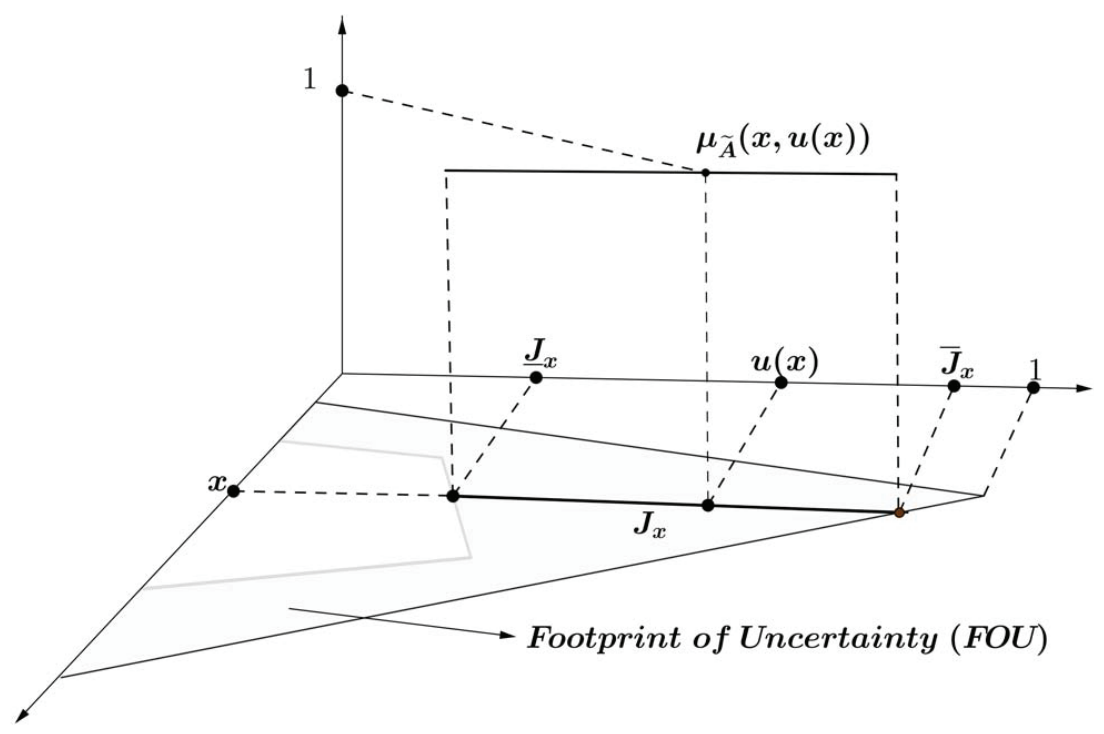

Figure 2: An interval type-2 fuzzy set.

Figure 2 shows the interval $J_{x}=\left[\underline{J}_{x}, \bar{J}_{x}\right] \subset[0,1]$ that determines two functions, $\underline{\mu}_{\widetilde{A}}(x)$, the lower membership, and $\bar{\mu}_{\widetilde{A}}(x)$ the upper membership, associated to the interval type- 2 fuzzy set as follows:

$$
\bar{\mu}_{\widetilde{A}}(x)=\bar{J}_{x}, \text { and } \underline{\mu}_{\widetilde{A}}(x)=\underline{J}_{x} .
$$


Back to the Figure 2, the Footprint of Uncertainty (FOU) is a region of the plane enclosed by the lower and upper membership functions. More precisely, we have

$$
F O U(\widetilde{A})=\bigcup_{x \in X}\left(x, J_{x}\right),
$$

where $\bigcup$ represents the union of sets in the classic sense.

In this work, the interval type-2 fuzzy set are use with the aim of comparison with type-1 fuzzy sets. As a model we use the population growth of a community, using as a technique the FRBS of both types.

\section{VERHULST'S MODEL WITH FUZZY PARAMETERS OF TYPE 1 AND 2}

The classic model of Verhulst or logistic model is given by the equations:

$$
\left\{\begin{array}{l}
\frac{d P}{d t}=r P\left(1-\frac{P}{P_{\infty}}\right) \\
P(0)=P_{0},
\end{array}\right.
$$

where $P(t)$ represents a population of individuals at the time $t, r$ is the reproduction rate of that particular population $(r>0)$ and $P_{\infty}$ is the capacity of support of the population.

The analytic solution of (2.1) is giving by:

$$
P(t)=\frac{P_{\infty} P_{0}}{\left(P_{\infty}-P_{0}\right) e^{-r t}+P_{0}} .
$$

In this study we consider the reproductive rate $r$ as being a value that depends on the fertility and economic development rates of the country.

These rates are the input variables of the FBRS in both cases. The output variable is the reproductive rate of the group studied.

To build the membership functions of the fertility and economic development rates, we gathered in a single FRBS the information from various data research institutes. As these institutes use different methodologies, the gradual information is compacted in a type-2 fuzzy sets. This way it brings together these gradualness, with values between the upper and lower membership functions, obtaining the FOU which includes all the information received.

We introduce two types of FBRS:

1. FBRS of type- 1 where the input and output linguistic variables are type- 1 fuzzy sets. The inference method use is the Mamdani's method [3], and the defuzzification method is the gravity center.

2. FBRS of type-2 where the input linguistic variables are interval type-2 fuzzy sets. The output variable is an interval. This system includes five components: fuzzifier, inference, rule base, type reducer, and defuzzifier. Figure 3 shows the structure of a type-2 FBRS. 


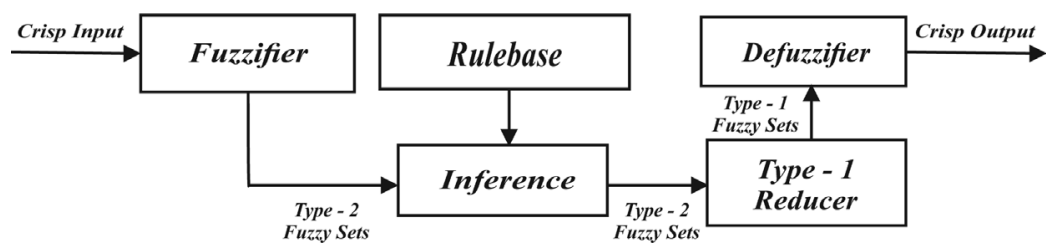

Figure 3: A Type-2 Fuzzy Rule-Based System.

In next subsection we are going to describe with more details the type-2 FBRS when the output is an interval.

\subsection{The Type-2 FBRS with Interval Output}

The content of this subsection can be checked with detail in [7]. One of the concepts used in this subsection from the fuzzy sets theory is the $t$-norm operator. A function $t$ from $[0,1] \times[0,1]$ into $[0,1]$ that is commutative, associative, and monotone with boundary conditions $0 t x=0$ and $1 t x=x$ is called a $t-$ norm.

Consider a type-2 SBRF with interval output, $Y^{n}=\left[\underline{y}^{n}, \bar{y}^{n}\right]$, and the rules $R^{n}$ define by

$$
\text { If } x_{1} \text { is } \widetilde{X}_{1}^{n} \text { and } x_{2} \text { and } \widetilde{X}_{2}^{n} \text { and } \cdots \text { and } x_{I} \text { is } \widetilde{X}_{I}^{n} \text { then } y \text { is } Y^{n}, n=1,2, \cdots, N \text {, }
$$

where $\widetilde{X}_{i}^{n}, i=1,2, \cdots, I$ are interval type-2 fuzzy sets. Being the input vetor of the form $x^{\prime}=$ $\left(x_{1}^{\prime}, x_{2}^{\prime}, \cdots, x_{I}^{\prime}\right)$, the steps to obtain the last component of the type-2 SBRF are the following [7]:

1. Compute the membership of $x_{i}^{\prime}$ at each $\widetilde{X}_{i}^{n}$, $\left[\underline{\mu}_{\tilde{X}_{i}^{n}}\left(x_{i}^{\prime}\right), \bar{\mu}_{\widetilde{X}_{i}^{n}}\left(x_{i}^{\prime}\right)\right], i=1,2, \cdots, I, n=$ $1,2, \cdots, N$;

2. Compute the initial interval of the $n^{\text {th }}$ rule, $F^{n}\left(x^{\prime}\right)=\left[\underline{f}^{n}, \bar{f}^{n}\right]$, as follows:

$$
\underline{f}^{n}=\underline{\mu}_{\widetilde{X}_{1}^{n}}\left(x_{1}^{\prime}\right) t \cdots t \underline{\mu}_{\widetilde{X}_{I}^{n}}\left(x_{I}^{\prime}\right), n=1,2, \cdots, N, i=1,2, \cdots, I,
$$

and

$$
\bar{f}^{n}=\bar{\mu}_{\widetilde{X}_{1}^{n}}\left(x_{1}^{\prime}\right) t \cdots t \bar{\mu}_{\widetilde{X}_{I}^{n}}\left(x_{I}^{\prime}\right), n=1,2, \cdots, N,
$$

where $t$ is a $t$-norm operator, $\underline{\mu}_{\widetilde{X}_{i}^{n}}\left(x_{i}^{\prime}\right)$ and $\bar{\mu}_{\widetilde{X}_{i}^{n}}\left(x_{i}^{\prime}\right)$, are the inferior and superior memberships of the corresponding type-2 input variables;

3. Perform what is called the type reduction method. There are many methods to do it and all of them have the purpose of combine $F^{n}\left(x^{\prime}\right)$ with the corresponding rule consequent. The most commonly of these methods, which is a generalized form of the type- 1 fuzzy sets method of the center of gravity, yields in a interval $\left[y_{l}, y_{r}\right]$ where

$$
y_{l}=\min _{k \in[1, N-1]} \frac{\sum_{n=1}^{k} \bar{f}^{n} \underline{y}^{n}+\sum_{n=k+1}^{N} \underline{f}^{n} \underline{y}^{n}}{\sum_{n=1}^{k} \bar{f}^{n}+\sum_{n=k+1}^{N} \underline{f}^{n}} \equiv \frac{\sum_{n=1}^{L} \bar{f}^{n} \underline{y}^{n}+\sum_{n=L+1}^{N} \underline{f}^{n} \underline{y}^{n}}{\sum_{n=1}^{L} \underline{f}^{n}+\sum_{n=L+1}^{N} \bar{f}^{n}},
$$


and

$$
y_{r}=\max _{k \in[1, N-1]} \frac{\sum_{n=1}^{k} \underline{f}^{n} \bar{y}^{n}+\sum_{n=k+1}^{N} \bar{f}^{n} \bar{y}^{n}}{\sum_{n=1}^{k} \underline{f}^{n}+\sum_{n=k+1}^{N} \bar{f}^{n}} \equiv \frac{\sum_{n=1}^{R} \underline{f}^{n} \bar{y}^{n}+\sum_{n=R+1}^{N} \bar{f}^{n} \bar{y}^{n}}{\sum_{n=1}^{R} \underline{f}^{n}+\sum_{n=R+1}^{N} \bar{f}^{n}},
$$

being $L$ and $R$ the so call switch points. These points are determined by

$$
\underline{y}_{L} \leq y_{l} \leq \underline{y}^{L+1}, \text { and } \bar{y}_{R} \leq y_{l} \leq \bar{y}^{R+1},
$$

where the sequences, $\underline{y}^{n}$ and $\bar{y}^{n}$, have been sorted in ascending order.

In this work the points $y_{l}$ and $y_{r}$ are computed using the Karnik-Mendel (KM) and a code provides by Dongrui Wu [7].

\section{RESULTS}

To obtain the solutions of the Verhuslt's model, we use the linguistics terms for the fertility and economic development rates of the country are, in both cases, low, medium, and high. In the case of type-1 FBRS it was used the same linguistic terms for the reproductive rate.

After some comparisons between the membership functions of type- 1 and type- 2 we concluded that, when all the lower membership functions are null in at least one of the input variables, then the defuzzified output surface is constant, returning a unique value for the reproductive rate, in our simulation, $r=0.5$. In the case that none of the input variable has this feature, the region of the range of the solutions does not present large variation between the maximal and minimal reproductive rates.

The input membership functions variables in both methods of the FBRS are shown in Figure 4.

Figure 5 shows the case that one of the input variables has all lower membership functions null.

The membership functions of the type-1 output variable are shown in Figure 6.

Observing the points of the universe where the membership degrees of the output variable of the FBRS of type-1 are non negative, it has been built the intervals of output variable for the FBRS of type-2, which are exactly the correspondents to those supports. Therefore, the nine fuzzy rules are shown in the Tables 1 and 2, where $x_{1}$ represents the variable "rate of fertility" and $x_{2}$ the variable "rate of (economic) development of the country".

In the following, we show the numerical simulations to compare the FBRS of both types obtaining the defuzzified surfaces and we plot the range of the maximal and minimal solutions correspondents to each method.

It has been confirmed the expectation that the region determined by the solutions correspondent to the minimal and maximal rate generated by the simulations of the type-1 FBRS "overlay" the correspondent region generated by the type-2 FBRS, confirming results on the literature [1], [2], [4] and [7], thus moving the better efficiency of the type-2 FBRS in acquirement information. 


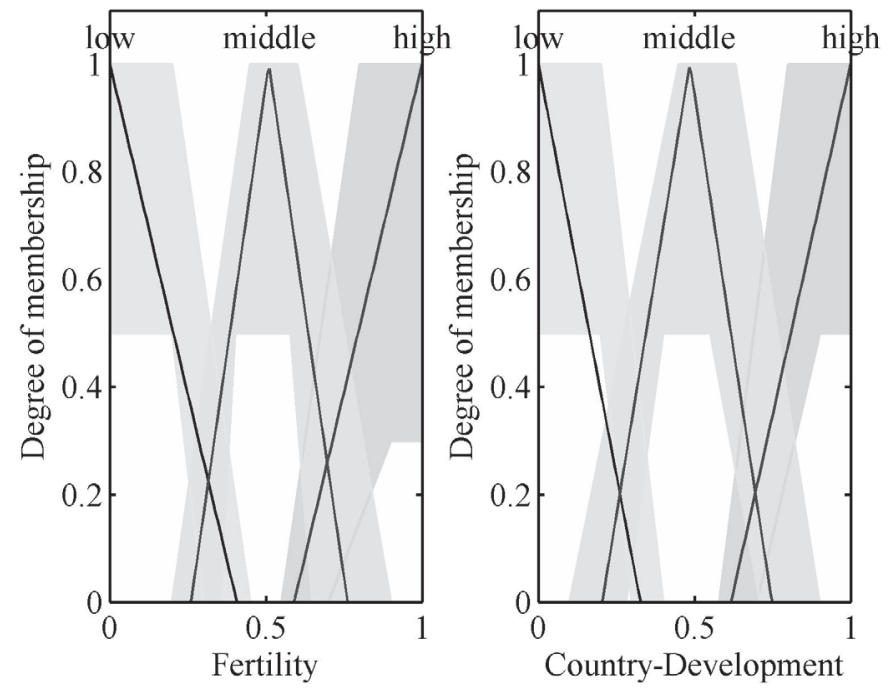

Figure 4: The input membership functions of type-1, in lines of dark gray color and those of type-2 shown in regions of gray light and dark color.
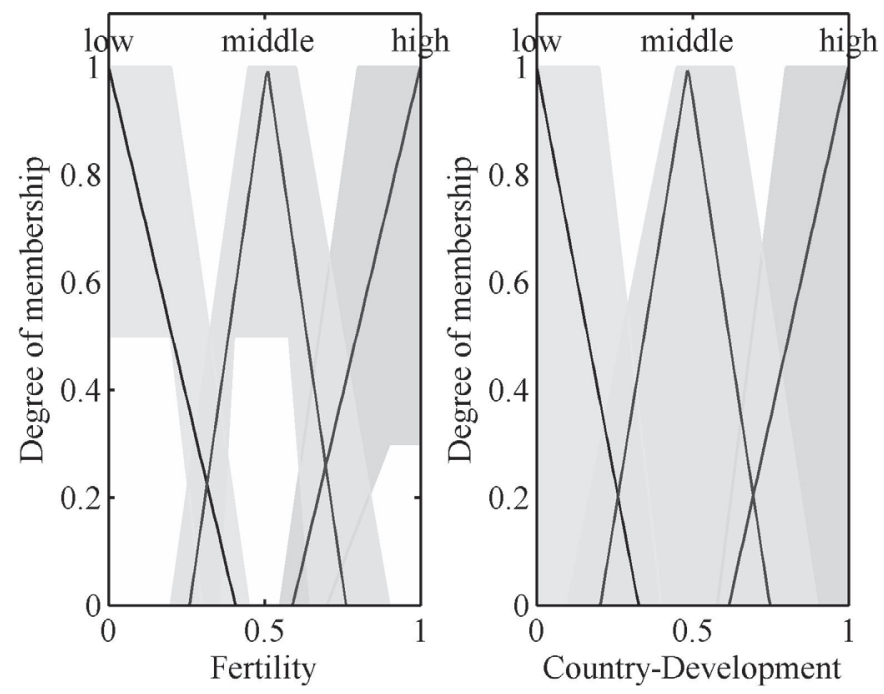

Figure 5: Input membership functions correspondent to the case that one of the input variables has null lower membership functions. The gray color scale follows the pattern of Figure 4.

Furthermore, when the membership functions are identically zero in at least one of the input variables, we observe that the defuzzified surface is a plane, representing the unique value of the reproductive rate. The defuzzified surfaces obtained from the type- 2 and type- 1 FBRS and are compared in the Figure 7 and 8, and in both cases the input variables are the correspondents in the Figures 4 and 5, respectively. 


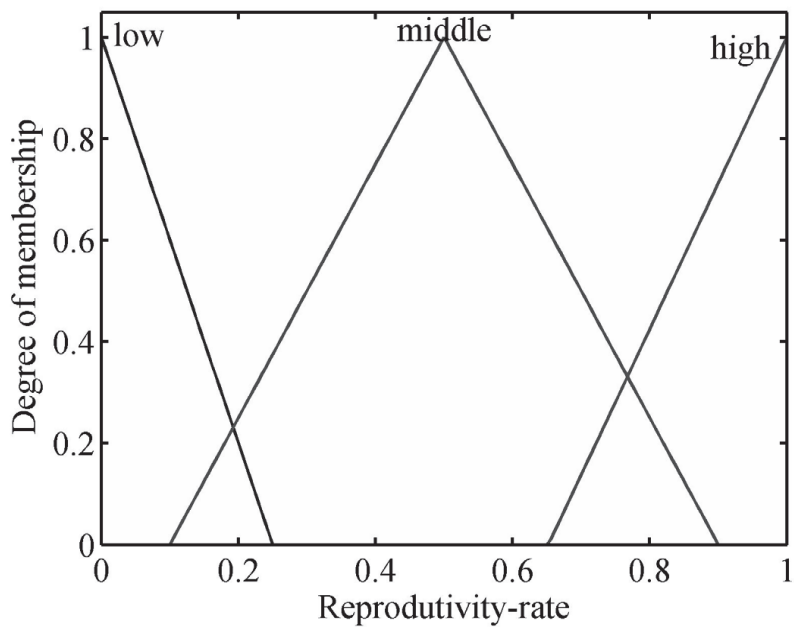

Figure 6: Membership functions of the type-1 output variable.

Table 1: The nine rules of the type-1 FBRS.

\begin{tabular}{|c|c|c|c|}
\hline$x_{1}$ & Low & Medium & High \\
\hline Low & Low & Low & Low \\
Medium & Medium & Medium & Low \\
High & High & High & Medium \\
\hline
\end{tabular}

Table 2: The nine rules of the type-2 FBRS.

\begin{tabular}{|c|c|c|c|}
\hline$x_{1} x_{2}$ & Low & Medium & High \\
\hline Low & {$\left[\begin{array}{ll}0 & 0.25\end{array}\right]$} & {$\left[\begin{array}{ll}0 & 0.25\end{array}\right]$} & {$\left[\begin{array}{ll}0 & 0.25\end{array}\right]$} \\
Medium & {$\left[\begin{array}{lll}0.1 & 0.9\end{array}\right]$} & {$\left[\begin{array}{ll}0.1 & 0.9\end{array}\right]$} & {$\left[\begin{array}{ll}0 & 0.25\end{array}\right]$} \\
High & {$\left[\begin{array}{lll}0.652 & 1\end{array}\right]$} & {$\left[\begin{array}{lll}0.652 & 1\end{array}\right]$} & {$\left[\begin{array}{ll}0.1 & 0.9\end{array}\right]$} \\
\hline
\end{tabular}

As an analytic solution of (2.1) giving by (2.2) is an increasing function with respect to $r$ for all $t$, all the solutions determined by the values obtained by $r$ are in between of the solutions $P_{M}(t)$ and $P_{m}(t)$, determined by the maximal $M$ and minimal $m$, respectively. Therefore, all the solutions are contained in a region bounded by $P_{m}(t)$ and $P_{M}(t)$, as shown in Figure 9 . In the case of the type-2 FBRS and using membership functions as the correspondents to Figure 5, the region reduces to a curve since the extremes $P_{M}(t)$ and $P_{m}(t)$ are equals for all $t$. This case is shown in Figure 10. 


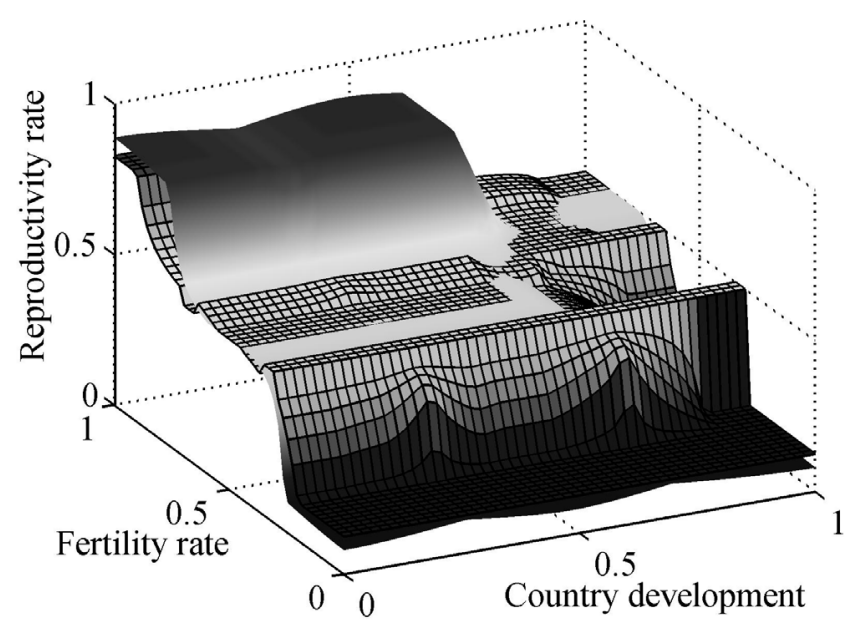

Figure 7: Comparison of the defuzzified surfaces from type-2 FRBS built with the KM algorithm (in "grid" mode) and the type-1 (in "shading" mode). The defuzzified surface from type-2 FRBS unfolds into a plane when the memberships of one input variable is null.

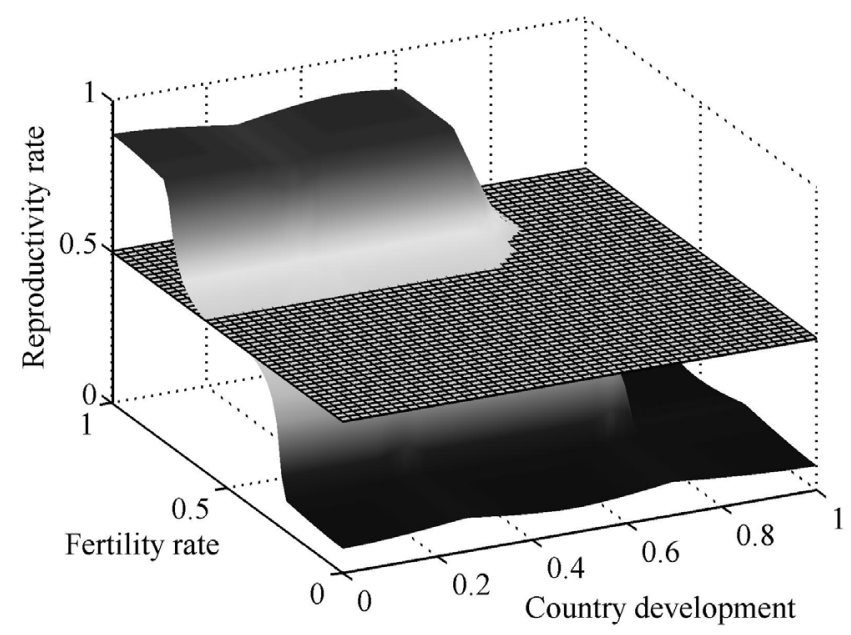

Figure 8: The defuzzified surface type-2 FRBS unfolds into a plane with the memberships of one input variable is null (see Fig. 5).

\section{CONCLUSIONS}

Recently the type-2 FBRS using the type reducer method has been shown a better approximation capacity than the correspondent to type- 1 through numerical experiments as confirmed by $\mathrm{Wu}$ and Nie [7]. Inspired by those affirmations, it was developed a comparative study of the type-1 and type-2 fuzzy rule-based system applied to the Verhulst model, where the reproductive rate 


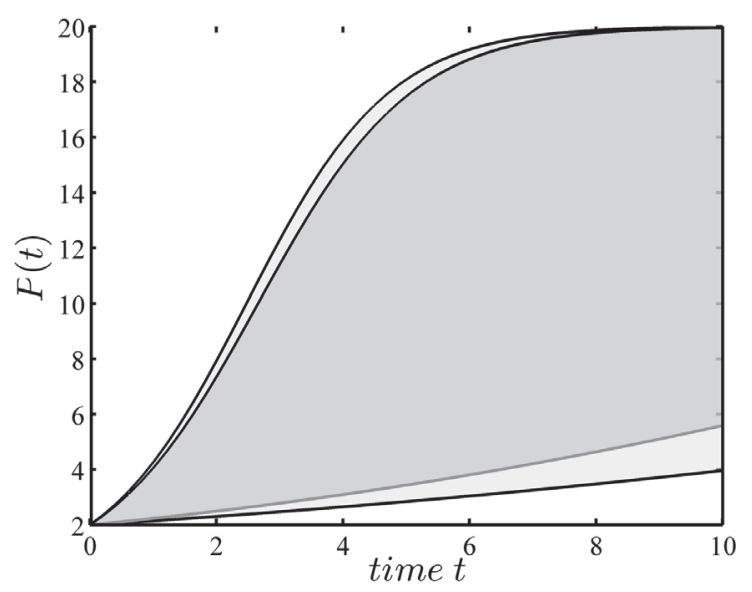

Figure 9: The region determined by the extremes of the solutions of the Verhulst's model for the reproduction rates obtained by both methods: in light gray color the generated by the type-1 FBRS and in dark gray the correspondents to type- 2 .

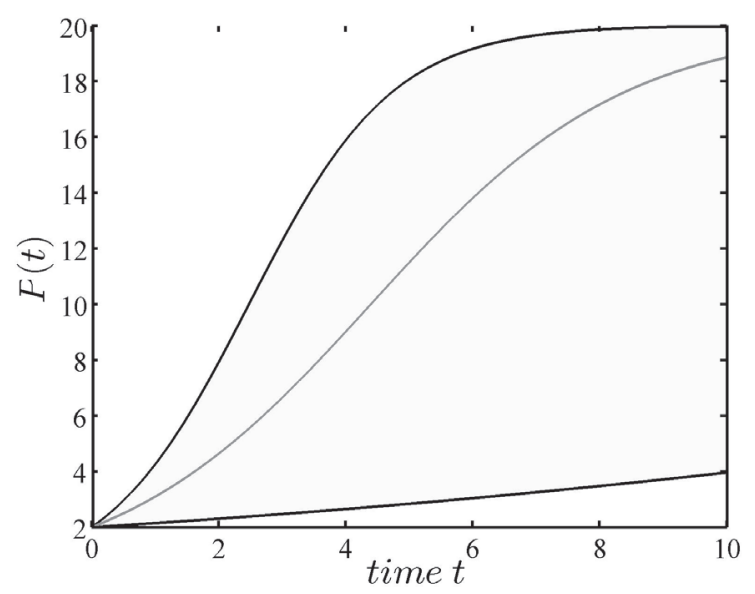

Figure 10: The case of Figure 5 has as correspondent regions the light gray color generated by the type-1 FBRS method and the unique solution in dark gray, the one generated by the type- 2 FBRS method.

is considerate as a type-1 fuzzy number of and interval type-2. As in type- 2 sets the FOU is characterized, we can incorporate the variability of the expert's knowledge into a unique FRBS in the population study. As a conclusion of the comparison of both methods, it has been observed that the output of the type-2 FBRS is more precise in the sense that the region of variation of the solutions of Verhulst's model is included in the region of the solutions generated by the type-1 fuzzy system, which shows a better precision in the response. We have observed an extreme case of a unique value for the reproductive rate in the case that the membership function of the one of the input variables are null. 
We believe that the present study will bring important information concerning the reason of the statement done in respect to the efficiency of type-2 fuzzy set to Verhuslt's model. Furthermore, we have the intention of continue to explores in another models of differential equations, ordinary or partial type, using the type-2 fuzzy sets. We are looking forward to obtain better results in the sense of precision that those obtained with the type-1 fuzzy set.

RESUMO. Este estudo apresenta o modelo do Verhulst para a análise do crescimento da população com a taxa de reprodutibilidade, dependendo da taxa de fecundidade e do desenvolvimento econômico do país. Estas variáveis linguísticas são definidas através de sistemas baseados em regras fuzzy (SBRF). A análise é feita para SBRF tipos 1 e 2 em que, no primeiro caso, o método usado é a inferência de Mamdani e a defuzificação é o centro de gravidade. Para o tipo 2 SBRF é usado como variáveis de entrada dos conjuntos fuzzy do tipo 2 intervalar, e intervalos como os de saída. A saída é defuzzificada pelo método de Redutor tipo que usa o algoritmo de Karnik-Mendel (KM). O objetivo deste estudo é comparar as soluções do modelo de Verhulst, onde o parâmetro, a taxa de reprodutibilidade, é determinado através das SBRF tipo 1 e tipo 2. A comparação é feita computando as regiões construída a partir das soluções correspondentes à taxa máxima e mínima. Foi notado que a região correspondente ao tipo-2 SBRF está contida na região construído de forma semelhante a partir de tipo $1 \mathrm{SBRF}$, mostrando uma maior precisão na resposta [1], [2] e [4].

Palavras-chave: Conjuntos fuzzy, sistemas baseado em regras fuzzy, equações diferenciais ordinárias.

\section{REFERENCES}

[1] S. Coupland \& R.I. John. Geometric type-1 and type-2 fuzzy logic systems. IEEE Trans. on Fuzzy Systems, 15(1) (2007), 3-15.

[2] H. Hagras. Type-2 FLCs: A new generation of fuzzy controllers. IEEE Computational Intelligence Magazine, 2(1) (2007), 30-43.

[3] E.H. Mamdani \& S. Assilian. An experiment in linguistic synthesis with fuzzy logic controller. Intern. J. of Man-machine Studies, 7(1) (1975), 1-13.

[4] N.N. Karnik, J.M. Mendel \& Q. Liang. Type-2 fuzzy logic systems. IEEE Trans. on Fuzzy Systems, 7 ((1999), 643-658.

[5] N.N. Karnik \& J.M. Mendel. Centroid of a type-2 fuzzy set. Information Sciences, 132 (2001), 195-220.

[6] J.M. Mendel. "Uncertain Rule-Based Fuzzy Logic Systems: Introduction and New Directions", Prentice-Hall, Upper-Saddle River, NJ (2001).

[7] D. Wu \& M. Nie. Comparison and practical implementation of type-reduction algorithms for type-2 fuzzy sets and systems. Proc. IEEE Intl Conf. on Fuzzy Systems, Taipei, Taiwan (2011).

[8] L.A. Zadeh. The concept of a linguistic variable and its application to approximate reasoning-1. Inform. Sci., 8 (1975), 199-249. 